\title{
Dopamine measurement during prolonged deep brain stimulation: a proof-of-principle study of paired pulse voltammetry
}

Seungleal (Brian) Paek, B.S. ${ }^{1}$, Emily Jane Knight, B.S. ${ }^{1}$, Su-Youne Chang, Ph.D. ${ }^{1,2}$, J. Luis Lujan, Ph.D. ${ }^{1}$, Dong Pyo Jang, Ph.D. ${ }^{3}$, Kevin E. Bennet, MBA ${ }^{4}$, and Kendall H. Lee, M.D., Ph.D. ${ }^{1,2}$

${ }^{1}$ Department of Neurosurgery, Mayo Clinic, Rochester, MN, United States of America 55905

${ }^{2}$ Department of Physiology and Biomedical Engineering, Mayo Clinic, Rochester, MN, United States of America 55905

${ }^{3}$ Department of Biomedical Engineering, Hanyang University, Seoul, South Korea

${ }^{4}$ Division of Engineering, Mayo Clinic, Rochester, MN, United States of America 55905

\begin{abstract}
Purpose-Deep Brain Stimulation (DBS) has been effective in treating various neurological and psychiatric disorders; however, its underlying mechanism hasn't been completely understood. Fast scan cyclic voltammetry (FSCV) is a valuable tool to elucidate underlying neurotransmitter mechanisms of DBS, due to its sub-second temporal resolution and direct identification of analytes. However, since DBS-like high frequency stimulation evokes neurotransmitter release as well as extracellular $\mathrm{pH}$ shift, it is hard to isolate the neurotransmitter signal from the complex environment. Here we demonstrate the efficacy of a modified FSCV technique, Paired Pulse Voltammetry (PPV), in detecting dopamine (DA) release in the caudate nucleus during long-term electrical stimulation of the medial forebrain bundle (MFB) in the rat.
\end{abstract}

Methods-Unlike traditional FSCV applying a single triangular waveform, PPV employs a binary waveform with a specific time gap $(2.2 \mathrm{~ms})$ in between the comprising pulses. DA measurement was performed with a carbon fiber microelectrode placed in the caudate nucleus and a twisted bipolar stimulating electrode in the MFB. PPV data was collected with the Wireless Instantaneous Neurochemical Concentration Sensing System (WINCS).

Results-Using PPV, the detection of DA was evident throughout the long-term stimulation (5 minutes); however, without PPV, in vivo environmental changes including $\mathrm{pH}$ shift eventually obscured the characteristic oxidation current of DA at $0.6 \mathrm{~V}$.

Conclusions-These results indicate that PPV can be a valuable tool to accurately determine DA dynamics in a complex in vivo environment during long-term electrical stimulation.

\section{Keywords}

Deep Brain Stimulation (DBS); Fast Scan Cyclic Voltammetry (FSCV); Paired Pulse Voltammetry (PPV); Dopamine (DA); Medial Forebrain Bundle (MFB)

Corresponding Author: Kendall H. Lee, M.D., Ph.D., Department of Neurologic Surgery and Department of Physiology and Biomedical Engineering, Mayo Clinic, 200 First Street SW, Rochester, Minnesota 55905, Telephone: 507-284-2816; Fax: 507-284-5206, lee.kendall@mayo.edu. 


\section{Introduction}

Deep Brain Stimulation (DBS) neurosurgery has now been widely performed throughout the world, achieving therapeutic success in various neurological and psychiatric conditions [1]. Among the most prominent disorders for which this treatment has proven effective are Parkinson's disease, essential tremor, dystonia, and recently obsessive compulsive disorder [2-8]. Despite the efficacy of DBS for a wide variety of conditions, the precise mechanism of its therapeutic action is still incompletely understood. However, techniques such as fast scan cyclic voltammetry (FSCV) have been emerging as valuable tools for mechanistic studies of DBS [9]. FSCV is an established electrochemical technique, which typically involves the application of a linearly varying electrical potential to a carbon fiber microelectrode (CFM) and measurement of the changes in current induced by the oxidation and reduction of neurochemicals. Using FSCV, neurochemicals can be differentiated from one another and the surrounding environment on the basis of the unique voltages at which they oxidize and reduce. This technique already has been utilized for both in vitro and in vivo neurochemical detection and if further refined has the potential to significantly advance our mechanistic understanding of DBS action [10-12]. Toward that end, since the inception of FSCV the sensitivity and specificity of in vivo neurochemical detection has been improved through the application of specific voltammetric waveforms [13-16]. However, these waveforms are as yet not optimally refined for in vivo settings, which often result in complex voltammograms, influenced by $\mathrm{pH}$ changes and other gross environmental changes at the CFM surface. To circumvent this limitation, data has only been collected over a short period of time following electrical or mechanical stimulation or advanced analysis algorithms such as principal component regression have been utilized [11]. Recently a modified FSCV technique termed paired pulse voltammetry (PPV) has been proposed as an alternative way to isolate neurotransmitters [17].

As compared to traditional FSCV, PPV can differentiate analytes based upon their adsorption characteristics, a unique property of each metabolite. This adsorption characteristic is determined in part by a metabolite's intrinsic properties such as molecular, size, mass, and charge, and is a decisive factor that affects the detection and sensitivity of the cyclic voltammetric response during FSCV on the microelectrode surface [17]. PPV takes advantage of these differences in adsorption through application of a binary voltammetric waveform, consisting of two identical pulses (primary and secondary) separated by a specific time gap with a negative holding potential [17]. Because the adsorption characteristics of neurotransmitters such as dopamine are distinct from that of other environmental factors, analytes can be discerned via subtraction of two backgroundsubtracted voltammograms.

Previously, PPV was tested in the flow cell with three different analytes (DA, adenosine, and $\mathrm{pH}$ changes), and it was found that the peak oxidation current decreased by approximately $50 \%, 80 \%$, and $4 \%$, respectively, in the secondary voltammogram as compared to the primary voltammogram. Thus, when the secondary voltammogram was subtracted from the primary voltammogram, $\mathrm{pH}$ influence was virtually eliminated [17]. In addition, this study demonstrated that PPV could detect increases in adenosine by mechanical disruption of cells in in vivo rat brains [17]. However, this prior study has not explored the efficacy of PPV for in vivo detection of neurochemical changes evoked by long-term stimulation such as that applied in the setting of DBS. Therefore to explore the efficacy of this technique for monitoring of neurochemicals during DBS, we performed PPV in in vivo rat brain during sustained stimulation. To do this, we used the Wireless Instantaneous Neurochemical Concentration Sensing System (WINCS) [1, 18] and Mayo Investigational Neuromodulation Control System (MINCS), in-house designed and fabricated wireless chemical sensing system and stimulator, respectively. The two devices 
can be synced via an optical connection to provide artifact-free measurements of stimulation-evoked neurochemical release.

We further chose to stimulate the medial forebrain bundle (MFB), an area of potential clinical importance, as MFB stimulation has recently been associated with psychiatric adverse events such as hypomania in STN DBS [19]. Because PPV has the potential to differentiate neurotransmitter release from complex environmental changes evoked by longterm stimulation to the MFB, it may prove a valuable technique to understand the neurotransmitter mechanisms underlying the therapeutic and adverse effects of stimulation to this and other targets. Thus, in the present study we tested the hypothesis that PPV would lead to improved sensitivity and specificity in the detection of DA evoked in the striatum by long-term electrical stimulation to the MFB in the rat.

\section{Materials and Methods}

\section{In Vivo surgery}

Six adult male Sprague-Dawley rats weighing 250-450g were used for this study. The rats were sheltered with a 12:12 hr light and dark cycle (lights on at $0600 \mathrm{hr}$ ) with ad libitum access to food and water. NIH guidelines were practiced for the care of animals and the Mayo Clinic Institutional Animal Care and Use Committee approved every experimental procedure.

The rats were injected with urethane (1.6 g/kg i.p.) for anesthesia and stabilized in a commercially available stereotaxic frame (David Kopf Instruments, Tujunga, CA, USA) for the surgery. Three burr holes $(0.5-1.0 \mathrm{~mm}$ diameter) were made on the skull of each rat for the implantation of a CFM, a twisted bioloar stimulating electrode (Plastics One, MS 303/2, Roanoke, VA, USA), and an $\mathrm{Ag} / \mathrm{AgCl}$ reference. Electrode coordinates were referenced by a rat brain atlas (Paxinos and Watson 1997) based on flat-skull position using bregma and dura as reference points with coordinates anteroposterior (AP), mediolateral (ML), and dorsoventral (DV). The CFM was placed in the right hemisphere in the dorsomedial striatum (AP $+1.2 \mathrm{~mm} ; \mathrm{ML}+2.0 \mathrm{~mm}$; DV -4.5 to $-6.0 \mathrm{~mm})$, and the stimulation electrode was inserted ipsilaterally just above the MFB (AP -4.6; ML +1.3; DV -8.0 to -9.0). The DV positions of both the stimulating electrode and CFM were optimized by electrically-evoked DA release in the striatum. Once optimized, the electrode locations were fixed for the remainder of the experiment. The reference electrode was positioned superficially in the cortical tissue of the left hemisphere.

\section{Electrochemical monitoring: FSCV and PPV}

Electrochemical recordings, including FSCV and PPV, were acquired by the WINCS system $[1,20]$. In brief, WINCS is a wireless, sterilizable, compact and low-noise device that incorporates front-end analog circuitry, a microrocessor, and Bluetooth radio, all on a single rechargeable lithium-polymer battery-powered, multiplayer, hermatically sealed printed circuit board, combining FSCV with digital telemetry to perform microsecond electrochemical measurements in the brain. PPV was applied at $5 \mathrm{~Hz}(200 \mathrm{~ms}$ between two binary pulse sweeps), $400 \mathrm{~V} / \mathrm{s}$ sweep rate, with respect to an $\mathrm{Ag} / \mathrm{AgCl}$ reference electrode, from $-0.4 \mathrm{~V}$ to $1.5 \mathrm{~V}$ back to $-0.4 \mathrm{~V}$, and $2.2 \mathrm{~ms}$ gap between the two pulses within a binary pulse sweep (Fig. 1). For each recording session, three different voltammograms were constructed. The primary voltammogram (P voltammogram) was produced with data at the primary pulse of the binary waveform; the secondary voltammogram ( $\mathrm{S}$ voltammogram) was produced with data at the secondary pulse of the waveform. Lastly, the difference voltammogram (P-S voltammogram) was built from subtracting the $\mathrm{S}$ voltammogram from the $\mathrm{P}$ voltammogram at each time point of data acquisition. Simultaneous collection of the 
three different voltammograms allowed us to gather both traditional FSCV data and PPV data in a same location of data acquisition. Background-subtracted cyclic voltammograms for the three types of voltammograms were calculated by subtracting each background current obtained concurrently.

\section{Carbon fiber microelectrodes (CFM)}

CFMs were fabricated by aspirating a single carbon fiber (Cytec Thornel T300) into a borosilicate glass capillary and pulling to a microscopic tip by a pipette puller (P-2000, Sutter Instrments, CA, USA). The exposed carbon fiber at the end of the tip was cut to a final length of $50 \mathrm{~mm}$. An $\mathrm{Ag} / \mathrm{AgCl}$ reference electrode was made by exposing $0.5 \mathrm{~mm}$ of a 31 gauge Teflon-coated silver wire (A-M Systems, Inc., Carlsborg, WA) to chlorine [21].

\section{Electrical stimulation}

Electrical stimulation was generated by the Mayo Investigational Neuromodulation Control System (MINCS), a recently developed wireless stimulation system, which synchronizes with the WINCS optically to produce artifact-free electrochemical recording during stimulation. MINCS integrates a 32-bit ARM-core mixed-signal microcontroller (Stellaris LM3S9B96, Texas Instruments), front-end analog circuitry for electrical stimulation, circuitry for sensing and fault protection, and a Bluetooth transceiver on a multiplayer printed circuit board, powered by a rechargeable, 6.5 watt-hour lithium-ion battery (Ultralife, Newark, NY, USA). The circuit board and battery are packaged into a plastic case. Electrical stimulation ( $100 \mathrm{uA}, 2 \mathrm{~ms}$ pulse width at $60 \mathrm{~Hz})$ was delivered continuously for 5 minutes.

\section{In vitro flow injection experiments}

Flow injection analysis was used to discern the $\mathrm{pH}$ effect on DA detection. This analysis system consisted of a FIAlab 3200 injection system (FIAlab Instruments, Seattle, WA) with an 8 port-switching valve. Measurements were taken as analyte was introduced as a bolus to a CFM positioned in a flowing stream of buffer $(150 \mathrm{mM}$ sodium chloride and $12 \mathrm{mM}$ Trizma base adjusted to $\mathrm{pH} 7.4$ ) at a rate of $2.25 \mathrm{ml} / \mathrm{min}$ using gravity feed. Solutions of 1 $\mu \mathrm{M}$ DA in buffer were prepared with varying $\mathrm{pH}$, ranging from $\mathrm{pH} 7.4$ to 7.8. All chemicals were purchased from Sigma Aldrich (Saint Louis, MO).

\section{Results}

\section{In-vivo detection of stimulation-evoked DA with PPV}

To detect in vivo dopamine release evoked by long-term MFB DBS (5 minutes), PPV was applied to a CFM that was stereotactically implanted into the striatum. DA was successfully detected by the P voltammogram, representative of data obtained with conventional FSCV, and by PPV (P-S voltammogram) (Fig. 2a and b, respectively). During the 5-minute stimulation, the oxidation peak current of DA was initially detected at $0.6 \mathrm{~V}$ in both FSCV techniques. However, the DA oxidation current as detected by conventional FSCV became obscured and eventually disappeared in 210 seconds of the stimulation, due to an appearance of unknown interfering molecule (Fig. 2a). This interfering molecule generated a negative current, which is the opposite direction of DA oxidation current, at $0.6 \mathrm{~V}$. This negative current confounds detection of the sustained DA oxidation current. In addition to this confounding reduction current at $0.6 \mathrm{~V}$, various changes were observed at other potentials; these changes did not directly affect the DA detection but may become problematic during detection of other chemicals such as adenosine, which is characterized by an oxidation potential at $1.5 \mathrm{~V}$. In contrast, PPV showed consistent DA oxidation current at $0.6 \mathrm{~V}$ until the stimulation terminated (Fig. 2b). 
Because the identity of analytes such as DA cannot be pharmacologically confirmed during clinical studies in humans, it is important to compare in vivo data with that obtained in controlled in vitro settings. For this reason, we compared the voltammograms obtained in vivo from the rat striatum with those obtained via injection of DA solutions in vitro in the flow cell. Notably, the reduction potential at $-0.2 \mathrm{~V}$, which is a component of the characteristic FSCV signature of DA (Fig. 3a), was not detected when PPV was applied both in vivo (Fig. 3b) and in vitro [17]. The loss of the reduction potential around $-0.2 \mathrm{~V}$ in the $\mathrm{P}$ $\mathrm{S}$ voltammogram is due to DA-o-quinone, a source of reduction current at the CFM surface that is not significantly influenced by the repetition time [17]. Additionally, the current induced by the same dopamine concentration is about $50 \%$ less in the P-S voltammogram as compared to the $\mathrm{P}$ voltammogram in the present study, also consistent with PPV experiments performed in vitro. Thus, DA absorption characteristics are similar and can be reliably detected even in the complicated in vivo environmental setting.

\section{In vivo coefficient of reaction time}

The reaction time constant ' $b$ ' for DA at $0.6 \mathrm{~V}$ was previously determined to be $14.5 \mathrm{~ms}$ in the in vitro setting to determine the optimum separation time between the two pulses [17]. To test if the same reaction time constant applies for the in vivo settings, we measured the DA peak oxidation current at $0.6 \mathrm{~V}$ of the secondary pulse (S voltammogram) using five different secondary pulse effective repetition time $(12.0,14.5,24.5,39.5,59.5,99.5 \mathrm{~ms}$, respectively) in three different rats. DA was measured after a short period of stimulation (2 sec) with the same parameter ( $100 \mathrm{uA}, 2 \mathrm{~ms}$ pulse width at $60 \mathrm{~Hz}$ ). After each measurement, 20 minutes rests were given until the next stimulation. Each in vivo data corresponds to the equation, $f(t)=a[1-\exp (-t / b)]$, using $14.5 \mathrm{~ms}$ as the reaction time constant ' $\mathrm{b}$ ' with Rsquared value of greater than 0.92 (Fig. $4 \mathrm{a}-\mathrm{c}$ ). This demonstrates that the optimum separation time determined in in vitro setting is applicable in in vivo environment as well.

\section{pH change and dopamine detection}

Next, we attempted to discern the nature of the confounding factors observed in the traditional FSCV voltammogram in vivo. It is well known that $\mathrm{pH}$ shift contributes to the complex voltammograms observed in vivo [22]. To investigate this $\mathrm{pH}$ effect, we compared the FSCV data collected in vivo with in vitro detection of DA in the presence of varying $\mathrm{pH}$ changes (basic) (Fig. 5). We observed that as the $\mathrm{pH}$ increased, the DA oxidation current at $0.6 \mathrm{~V}$ decreased gradually. In Fig. 5a, a red box on each of the color plots of the varying $\mathrm{pH}$ levels indicates this decrease in the oxidation current at $0.6 \mathrm{~V}$. Fig. $5 \mathrm{~b}$ shows the current vs. voltage plots for the varying $\mathrm{pH}$ changes, demonstrating gradual decrease in the current level detected at $0.6 \mathrm{~V}$. As the $\mathrm{pH}$ level became more basic, the positive currents at $-0.3 \mathrm{~V}$ and $+1.5 \mathrm{~V}$ were enhanced, as were the negative currents at $+0.45 \mathrm{~V}$ and $+0.7 \mathrm{~V}$, which eventually obscured the DA oxidation current at $0.6 \mathrm{~V}$ (Fig. $5 \mathrm{~b}$ ), similarly to the pattern observed in vivo (Fig. 2a-b).

Lastly, if neurochemical recording is to be performed in the human patient, there will be limited time for stabilization of the CFM during recording. Therefore, we tested the efficacy of PPV in reducing the initial dramatic background shift that is observed consistently at the initiation of changes in the environment surrounding the carbon tip of the electrode when time is not permitted for stabilization. We found that the PPV method can minimize the majority of the background drifting effect that is apparent when conventional FSCV is used. Fig. 6a shows the oxidation and reduction changes in the background surrounding the DA oxidation peak that were obtained via conventional FSCV with 1-second stimulation in vivo, obscuring the overall DA data. However, PPV minimizes the drifting effect, resulting in unblemished data that would be preferred in a fast-paced human study setting (Fig. 6b). 


\section{Discussion}

\section{Characteristic of DA release during long-term electrical stimulation}

Here, we report the successful use of PPV for DA measurement with WINCS during longterm DBS in the rat. This represents a significant advance, as previous FSCV measurements have been made only in a short period immediately following electrical stimulation. Detection of neurochemicals during longer stimulation is hampered by confounding factors, such as $\mathrm{pH}$ and other transient effects, that create non-specific large currents and obscure the neurochemical signatures. PPV can minimize these confounding events and provide an opportunity to scrutinize underlying neurochemicals. In our study, with PPV we unmasked continued DA oxidation current throughout 5-minute electrical stimulation whereas the DA oxidation current measured by conventional FSCV disappeared within 210 seconds. Therefore, we suggest that PPV will become a valuable tool for mechanistic studies of neurochemical release evoked by longer-term stimulation in in vivo models, more similar to DBS in clinical settings.

\section{Comparison of pH effects in vitro and in vivo}

Importantly, the nature of the confounding signals observed in voltammograms attempt to understand the complex in vivo electrochemical environment we systematically tested the possibility that $\mathrm{pH}$ shifts can account for some of the obfuscation of neurochemical signatures. When varying the $\mathrm{pH}$ level in the presence of DA in vitro we observed similar patterns as seen in vivo, including a reduction current occurring at around $0.5 \mathrm{~V}$, below the DA oxidation peak potential. In vitro, the reduction potential became more apparent as the $\mathrm{pH}$ level became more basic, also eventually obscuring the DA detection, as was seen with electrical stimulation in vivo. However, the results obtained by varying $\mathrm{pH}$ did not fully explain other patterns that were detected in the in vivo setting. From this we concluded that other factors in addition to $\mathrm{pH}$ change are likely responsible for the mixed signals obtained in vivo. Further work is needed to identify the nature of these factors, but PPV will likely prove to be an effective technique to unmask underlying stimulation-evoked neurochemicals in the face of a variety of obscuring signals that will likely occur in clinical studies of DBS.

\section{Indication of PPV Implementation}

Due to the complex environment characterized by $\mathrm{pH}$ changes and other gross environmental changes, sensing and discerning in vivo neurochemicals have proven to be difficult. To circumvent such a challenge, only the easily distinguishable electrochemical signal data during a brief time after either electrical or mechanical stimulation has been analyzed [11]. However this approach severely limits the experimental design, precluding longer stimulation effects on chronic in vivo neurochemical monitoring. To address this issue, advanced analysis algorithms such as principal component regression (PCR) have been successfully utilized to quantify dopamine and adenosine concentrations in complex voltammograms. [23-25] Also, PCR has successfully differentiated various analytes including $\mathrm{pH}$ in complex voltammograms in both in vitro and in vivo [24, 26-28]. Side by side with advanced post-processing techniques such as PCR, we proposed PPV with in vitro data acquisition, hoping to improve the real-time evaluation of neuronal metabolites of interest in compound electrochemical environments [17]. Here we further demonstrates PPV's potential with proof of principal in vivo data acquisition.

Importantly, PPV offers advantages over these post-processing techniques in that it can be performed during real-time analysis of the data. As a result, PPV is much better suited to human applications of FSCV in which real-time analysis is clearly preferred. 
However, PPV does have limitations in areas where PCR excels. For example, PCR analysis produces a statistical error measurement to establish the veracity of the analysis. By comparison, the technique of PPV heavily relies on a qualitative identification of the recorded signal. Also, for dopamine, approximately half of the oxidation signal at $+0.6 \mathrm{~V}$ is lost. Chemical resolution of the voltammogram is reduced by PPV eliminating other neurochemicals or ions that may be important factors. However, since PPV data is collected simultaneously with conventional FSCV data (P voltammogram), the full chemical resolution can be available for analysis. Lastly, the loss of DA reduction peak at $-0.2 \mathrm{~V}$ could be an issue. However, the loss of the reduction peak is not considered to be a significant issue as pre- and post-calibration are standards of practice to definitively confirm neurochemical identifies. In fact, these are performed even with traditional FSCV to eliminate any ambiguity in the data. For example, serotonin and dopamine can be confused on a traditional voltammogram and other techniques including microdialysis and drug studies can aid in resolution of these issues.

Thus, while PCR remains the definitive approach, PPV can provide an adequate visualization neurochemical of release to inform experimental decision on a rapid basis. Therefore, PPV does contribute to the FSCV studies of neurochemical release evoked by longer-term stimulation in in vivo models and for human recordings, where a fast-paced study setting is required to determine and qualify the neurochemicals of interest such as DA and ADO.

\section{Clinical Implications of MFB stimulation}

In addition to the clinical importance of this new technique, we have chosen to stimulate an area that may be involved in therapeutic or adverse effects of DBS in order to begin to explore the involvement of dopamine in these mechanisms. Therefore, in the present study, we stimulated the MFB, a structure of the reward circuit and a fundamental structure of the mesolimbic-dopamine system, which is implicated in affective disorders, drug addiction, and learning [29]. The MFB is a bundle of fibers that connect the ventral tegmental area (VTA), the lateral hypothalamus, ventral striatum, caudate nucleus, and septal area. Although these structures of reward and motivation have been studied extensively in the rodent [30-32], the underlying neurochemical mechanisms of long-term electrical stimulation similar to that performed in DBS for clinical applications have not been reported.

Interestingly, it has been recently suggested that MFB stimulation causes acute hypomania among advanced Parkinson's disease patients with inferiorly and medially located active electrode contacts in STN, in the proximity of the MFB. This represents a significant problem, as Tsai et al. [33] reported that among a group of 38 patients who underwent STN DBS about $13 \%$ of them showed symptoms of psychiatric adverse events (PAE) in the form of mania and hypomania during the first 3 months treatment. In fact, PAE or neuropsychological events after STN DBS have been reported to be as high as $41 \%$ for cognitive deficits, $8 \%$ for depression, and $4 \%$ for acute hypomania [33, 34]. Despite increasing recognition of PAE associated with electrodes in the proximity of the MFB, the neurochemical mechanism of such stimulation has not been thoroughly explored, largely due to difficulties in discerning neurotransmitters during long-term stimulation in vivo.

Symptoms of hypomania during the stimulation of the MFB can potentially be explained with the consistent release of DA in the striatum, such as that detected by PPV in the present study, as DA aberration is implicated in a variety of psychopathology, including manic symptomatology. This study represents a critical advance toward future studies to explore the relationship between the MFB stimulation-evoked striatal DA detected here and psychopathology in complex in vivo settings. Indeed, such a pattern of DA release during 
the stimulation could not have been discovered if the conventional FSCV method had been applied, as other confounding effects would have obscured accurate depiction of DA. Therefore this methodology lays the foundation for additional in vivo recordings employing stimulation parameters comparable to those utilized clinically in STN DBS as well as behavioral studies to investigate the relationship of MFB stimulation-evoked neurochemical release with hypomanic manifestations.

Overall, we have demonstrated the in vivo applicability of a novel technique for FSCV that will enable improvements in our ability to measure stimulation-evoked neurochemicals in animal models and human patients. Thus, this study provides a technique that will be instrumental in future studies to enhance the understanding of the relationship between neurotransmission and clinical outcome in DBS.

\section{Conclusions}

Here we introduce the effective application of PPV during in vivo detection of analytes in question that have previously been obscured by $\mathrm{pH}$ shift and other confounding effects during long-term stimulation similar to DBS. Therefore, PPV is a powerful tool to detect neurochemical release in situations where this was previously nearly impossible. PPV will be a useful technique to discern and measure stimulation-evoked neurochemicals in future preclinical and clinical studies.

\section{Acknowledgments}

This work was supported by: NIH (K08 NS 52252, R01 NS 70872 and R01 NS 74013 awards to KHL), Mayo Clinic Discovery Translation Fund, and The Grainger Foundation.

\section{References}

1. Bledsoe JM, Kimble CJ, Covey DP, Blaha CD, Agnesi F, Mohseni P, Whitlock S, Johnson DM, Horne A, Bennet KE, Lee KH, Garris PA. Development of the Wireless Instantaneous Neurotransmitter Concentration System for intraoperative neurochemical monitoring using fast-scan cyclic voltammetry. J Neurosurg. 2009; 111(4):712-23.

2. Benabid AL. Deep brain stimulation for Parkinson's disease. Curr Opin Neurobiol. 2003; 13(6):696706.

3. Greene P. Deep-brain stimulation for generalized dystonia. N Engl J Med. 2005; 352(5):498-500.

4. Hardesty DE, Sackeim HA. Deep brain stimulation in movement and psychiatric disorders. Biol Psychiatry. 2007; 61(7):831-5.

5. Limousin P, Krack P, Pollak P, Benazzouz A, Ardouin C, Hoffmann D, Benabid AL. Electrical stimulation of the subthalamic nucleus in advanced Parkinson's disease. N Engl J Med. 1998; 339(16):1105-11.

6. Mayberg HS, Lozano AM, Voon V, McNeely HE, Seminowicz D, Hamani C, Schwalb JM, Kennedy SH. Deep brain stimulation for treatment-resistant depression. Neuron. 2005; 45(5):65160.

7. Mazzone P, Lozano A, Stanzione P, Galati S, Scarnati E, Peppe A, Stefani A. Implantation of human pedunculopontine nucleus: a safe and clinically relevant target in Parkinson's disease. Neuroreport. 2005; 16(17):1877-81.

8. Volkmann J. Deep brain stimulation for the treatment of Parkinson's disease. J Clin Neurophysiol. 2004; 21(1):6-17.

9. Robinson DL, Hermans A, Seipel AT, Wightman RM. Monitoring rapid chemical communication in the brain. Chem Rev. 2008; 108(7):2554-84.

10. Garris PA. Advancing neurochemical monitoring. Nat Methods. 2010; 7(2):106-8. 
11. Heien ML, Khan AS, Ariansen JL, Cheer JF, Phillips PE, Wassum KM, Wightman RM. Real-time measurement of dopamine fluctuations after cocaine in the brain of behaving rats. Proc Natl Acad Sci U S A. 2005; 102(29):10023-8.

12. Borland, LM.; Michael, AC. An Introduction to Electrochemical Methods in Neuroscience. 2007.

13. Jackson BP, Dietz SM, Wightman RM. Fast-scan cyclic voltammetry of 5-hydroxytryptamine. Anal Chem. 1995; 67(6):1115-20.

14. Garris, PA.; Wightman, RM. Neuromethods: Voltammetric Methods in Brain Systems. Boulton, A.; Baker, G.; Adams, RN., editors. Totowa, NJ: Humana Press Inc; 1995.

15. Rice, ME.; Nicholson, C. Neuromethods:Voltammetric Methods in Brain Systems. Boulton, A.; Baker, G.; Adams, RN., editors. Totowa, NJ: Humana Press, Inc; 1995.

16. Heien ML, Phillips PE, Stuber GD, Sepel AT, Wightman RM. Overoxidation of carbon-fiber microelectrodes enhances dopamine adsorption and increases sensitivity. Analyst. 2003; 128(12): 1413-9.

17. Jang DP, Kim I, Chang SY, Min HK, Arora K, Marsh MP, Hwang SC, Kimble CJ, Bennet KE, Lee KH. Paired pulse voltammetry for differentiating complex analytes. Analyst. 2012; 137(6): 1428-35.

18. Griessenauer CJ, Chang SY, Tye SJ, Kimble CJ, Bennet KE, Garris PA, Lee KH. Wireless Instantaneous Neurotransmitter Concentration System: electrochemical monitoring of serotonin using fast-scan cyclic voltammetry--a proof-of-principle study. J Neurosurg. 2010; 113(3):656-65.

19. Coenen VA, Honey CR, Hurwitz T, Rahman AA, McMaster J, Bürgel U, Mädler B. Medial forebrain bundle stimulation as a pathophysiological mechanism for hypomania in subthalamic nucleus deep brain stimulation for Parkinson's disease. Neurosurgery. 2009; 64(6):1106-14. discussion 1114-5.

20. Shon YM, Chang SY, tye SJ, Kimble CJ, Bennet KE, Blaha CD, Lee KH. Comonitoring of adenosine and dopamine using the Wireless Instantaneous Neurotransmitter Concentration System: proof of principle. J Neurosurg. 2010; 112(3):539-48.

21. Garris PA, Christensen JR, Rebec GV, Wightman RM. Real-time measurement of electrically evoked extracellular dopamine in the striatum of freely moving rats. J Neurochem. 1997; 68(1): $152-61$.

22. Takmakov P, Zachek MK, Keithley RB, Bucher ES, McCarty GS, Wightman RM. Characterization of local $\mathrm{pH}$ changes in brain using fast-scan cyclic voltammetry with carbon microelectrodes. Anal Chem. 2010; 82(23):9892-900.

23. Swamy BE, Venton BJ. Carbon nanotube-modified microelectrodes for simultaneous detection of dopamine and serotonin in vivo. Analyst. 2007; 132(9):876-84.

24. Cechova S, Venton BJ. Transient adenosine efflux in the rat caudate-putamen. J Neurochem. 2008; 105(4):1253-63.

25. Cechova S, Elsobky AM, Venton BJ. A1 receptors self-regulate adenosine release in the striatum: evidence of autoreceptor characteristics. Neuroscience. 2010; 171(4):1006-15.

26. Heien ML, Johnson MA, Wightman RM. Resolving neurotransmitters detected by fast-scan cyclic voltammetry. Anal Chem. 2004; 76(19):5697-704.

27. Sanford AL, et al. Voltammetric detection of hydrogen peroxide at carbon fiber microelectrodes. Anal Chem. 2010; 82(12):5205-10.

28. Swamy BE, Venton BJ. Subsecond detection of physiological adenosine concentrations using fastscan cyclic voltammetry. Anal Chem. 2007; 79(2):744-50.

29. Panksepp, J. Affective neuroscience : the foundations of human and animal emotions. New York ; Oxford: Oxford University Press; 1998. p. xiip. 466

30. Carlezon WA Jr, Chartoff EH. Intracranial self-stimulation (ICSS) in rodents to study the neurobiology of motivation. Nat Protoc. 2007; 2(11):2987-95.

31. Wise RA. Addictive drugs and brain stimulation reward. Annu Rev Neurosci. 1996; 19:319-40.

32. Wise RA. Forebrain substrates of reward and motivation. J Comp Neurol. 2005; 493(1):115-21.

33. Tsai ST, et al. Neuropsychological effects after chronic subthalamic stimulation and the topography of the nucleus in Parkinson's disease. Neurosurgery. 2007; 61(5):E1024-9. discussion E1029-30. 
34. Temel Y, et al. Behavioural changes after bilateral subthalamic stimulation in advanced Parkinson disease: a systematic review. Parkinsonism Relat Disord. 2006; 12(5):265-72. 
a. Conventional FSCV

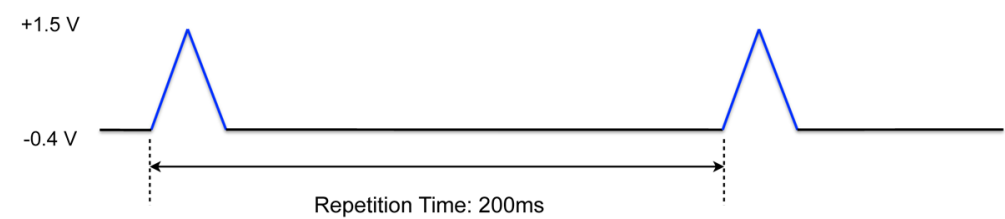

\section{b. Paired Pulse Voltammetry}

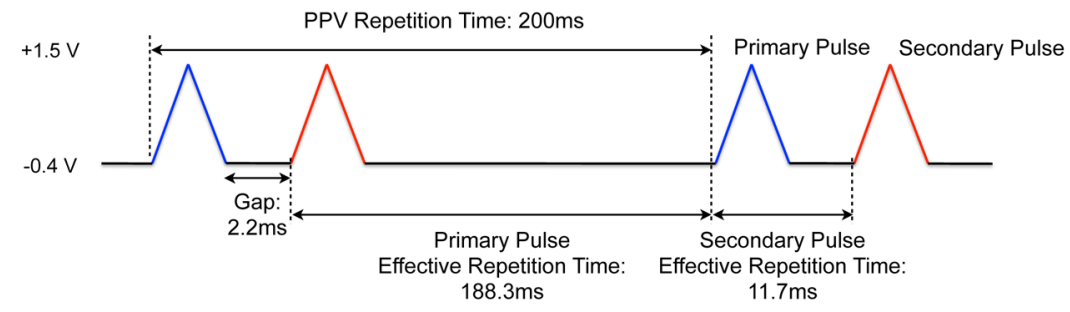

Fig 1.

(a)Schematic representation of conventional FSCV waveform for DA detection: a triangular shape waveform ramping from $-0.4 \mathrm{~V}$ to $1.5 \mathrm{~V}$, again back to -0.4 with a $200 \mathrm{~ms}$ repetition time. (b) Schematic representation of paired-pulse waveform for DA detection: two triangular waveforms are applied with a specific time gap in between and consistent holding potential. The effective repetition times of the primary pulse and the secondary pulse are set to be $188.3 \mathrm{~ms}$ and $11.7 \mathrm{~ms}$, respectively, with a $2.2 \mathrm{~ms}$ gap time. 


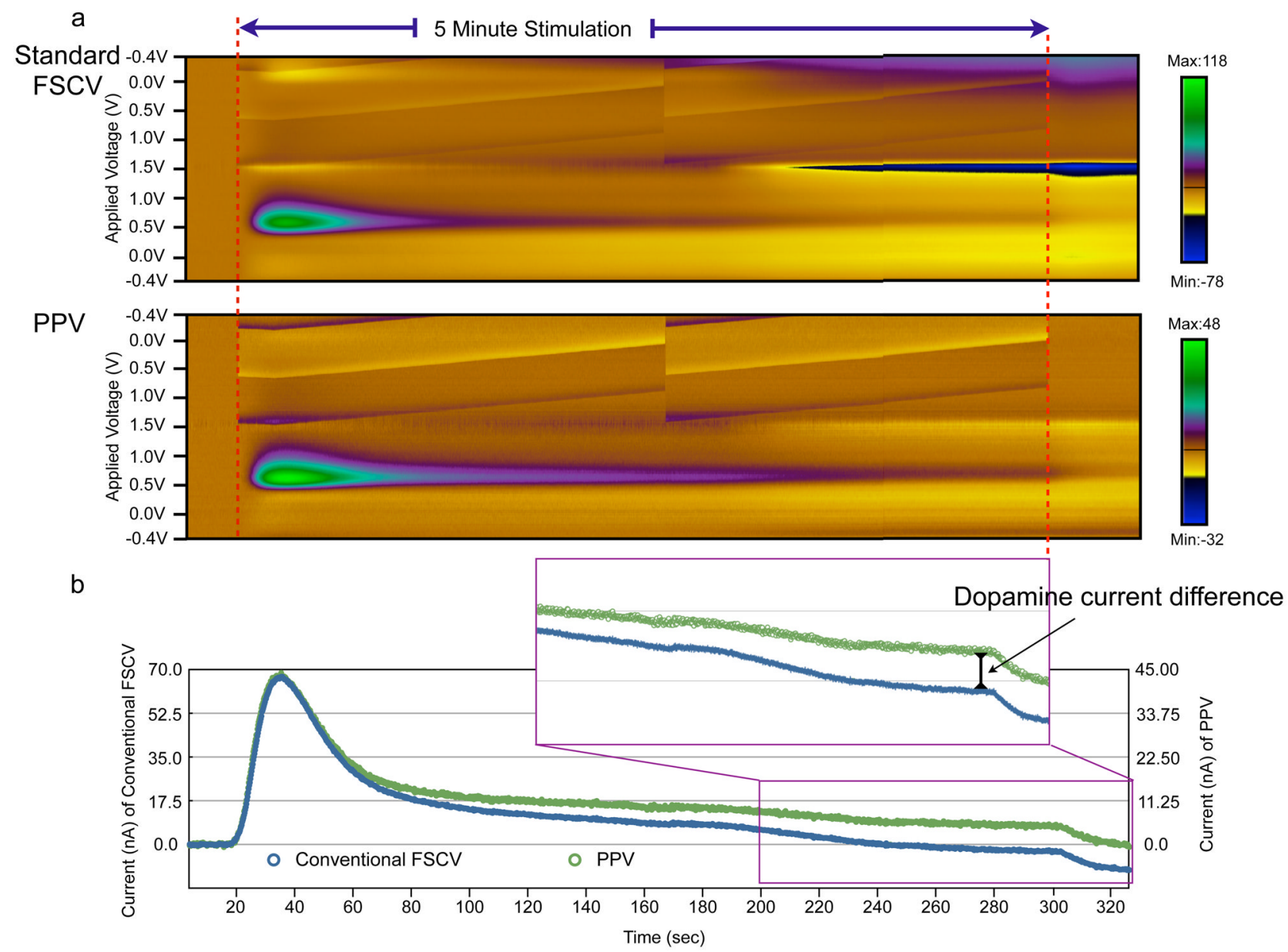

Fig 2.

(a) DA release evoked in the caudate by 5-minute electrical stimulation $(60 \mathrm{~Hz}, 2 \mathrm{~ms}$ Pulse Width, $100 \mathrm{uA}$ ) to the MFB as measured by traditional FSCV method (Top) and PPV (Bottom). (b) A plot of current (nA) vs. time comparing the patterns of DA release at $0.63 \mathrm{~V}$ in PPV (green) and conventional FSCV data (blue). 


\section{a. Conventional FSCV}

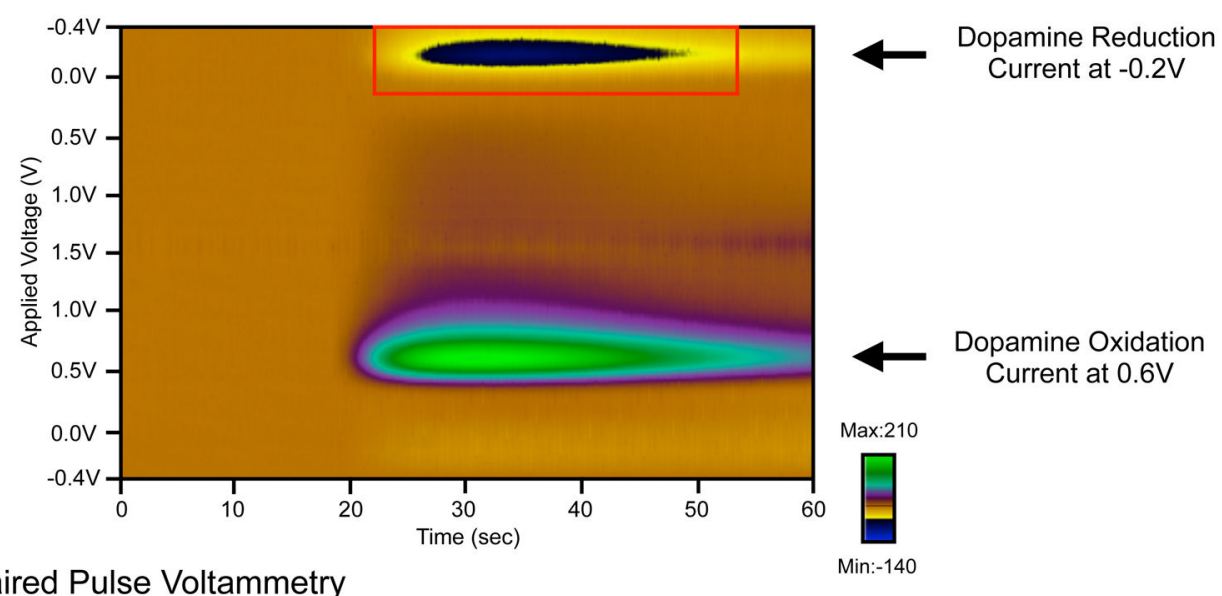

\section{b. Paired Pulse Voltammetry}

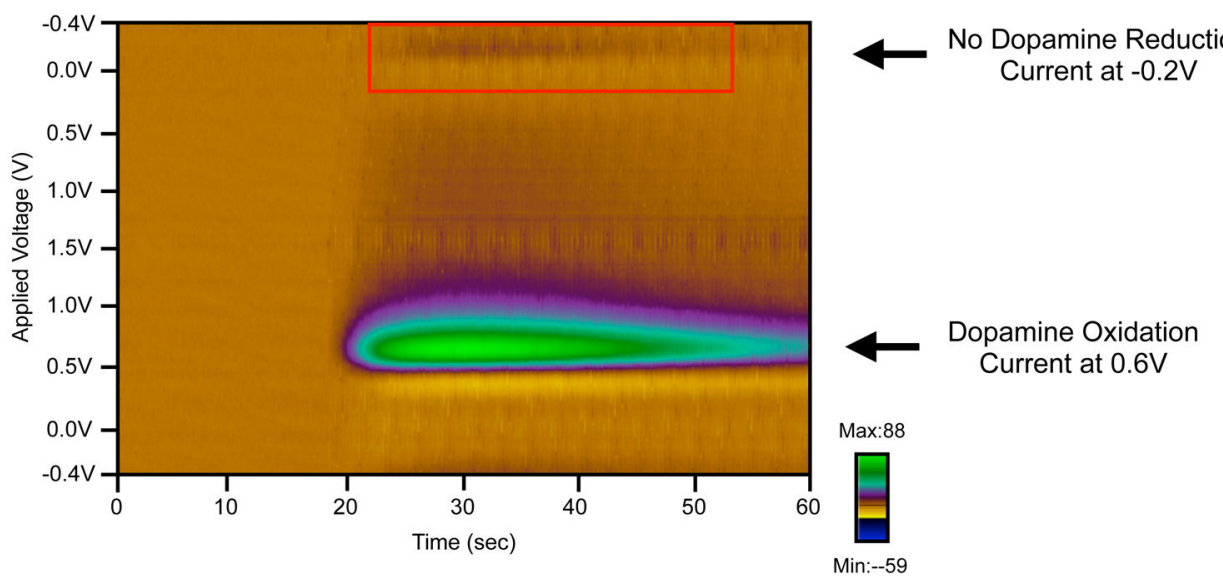

Fig 3.

A comparison of DA signature oxidation and reduction potentials, detected by conventional FSCV method and PPV method in vivo. (a) Pseudo-color plot of DA detected by conventional FSCV. The signature reduction potential at $-0.2 \mathrm{~V}$ and the oxidation potential at $0.6 \mathrm{~V}$ were both detected during electrical stimulation $(60 \mathrm{~Hz}, 2 \mathrm{~ms}$ Pulse Width, 100uA).

(b) Pseudo-color plot of DA detected by conventional FSCV. Only the oxidation potential at $0.6 \mathrm{~V}$ was detected during electrical stimulation $(60 \mathrm{~Hz}, 2 \mathrm{~ms}$ Pulse Width, $100 \mathrm{uA})$. 

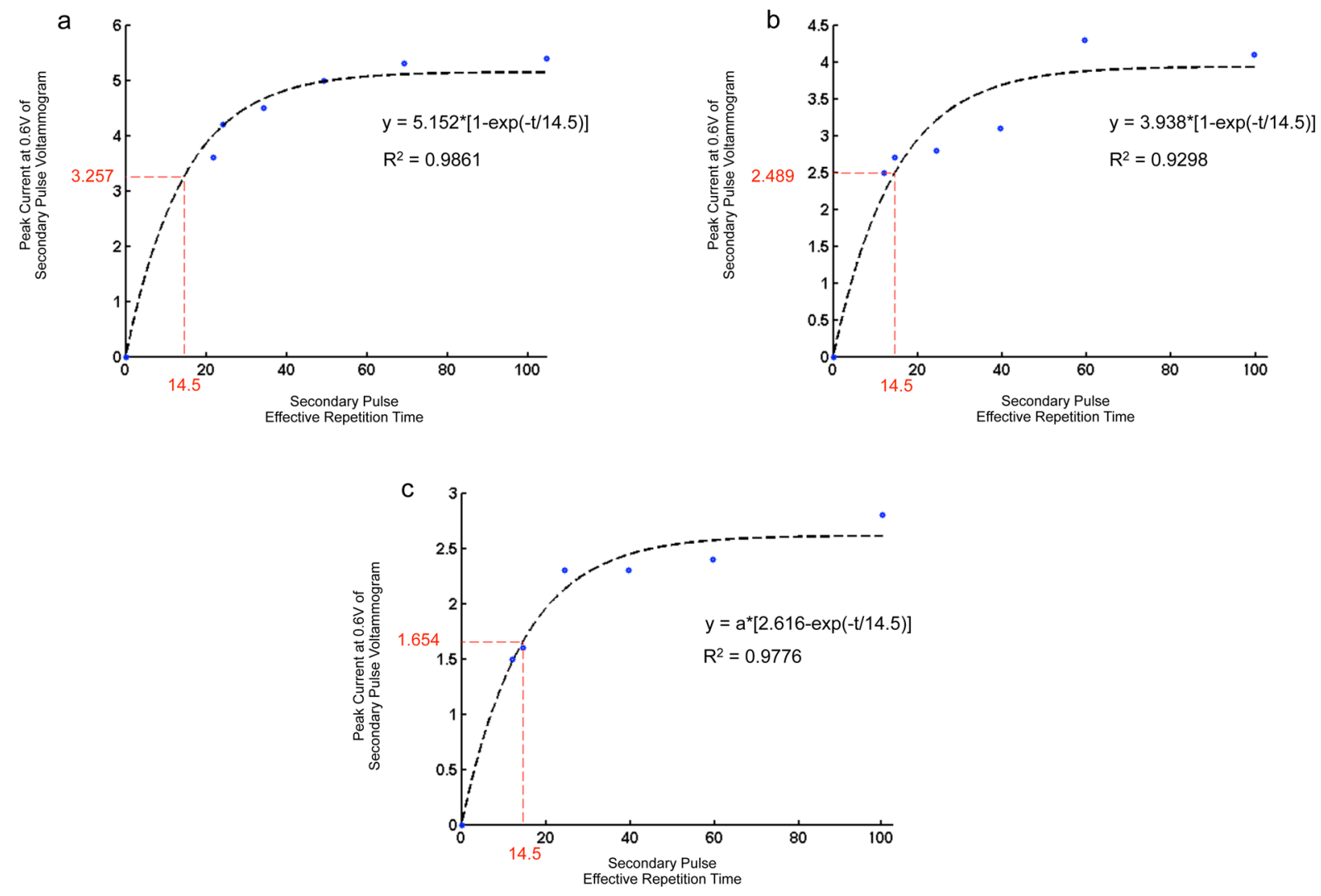

Fig 4.

The effect of repetition time on PPV in in vivo setting. Plot of DA peak currents at $0.6 \mathrm{~V}$ from the $\mathrm{S}$ voltammogram using five effective repetition times $(12.0,14.5,24.5,39.5,59.5$, $99.5 \mathrm{~ms}$ respectively) in three different rats. $(\mathrm{a}-\mathrm{c})$ The current $(\mathrm{nA})$ at $0.6 \mathrm{~V}$ for each effective repetition time, fitted with the exponential curve using the reaction time coefficient value of $14.5 \mathrm{~ms}$ within each rat ( $\mathrm{R}$-squared value $=0.9861,0.9298,0.9776$, respectively). 
a
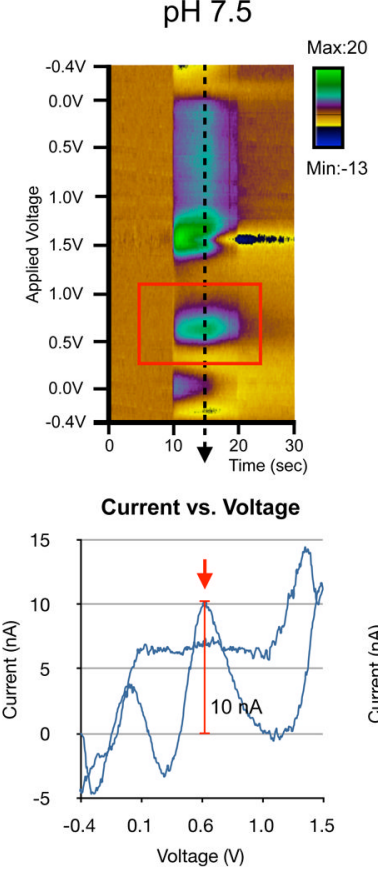

pH7.6

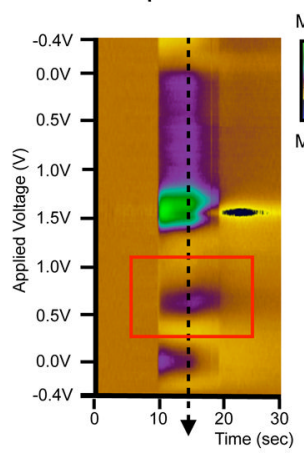

Current vs. Voltage

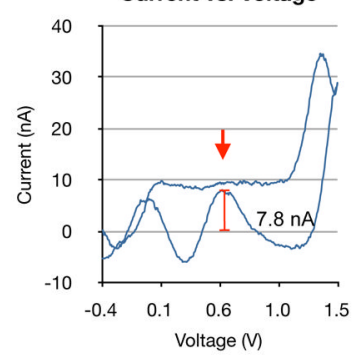

pH 7.7
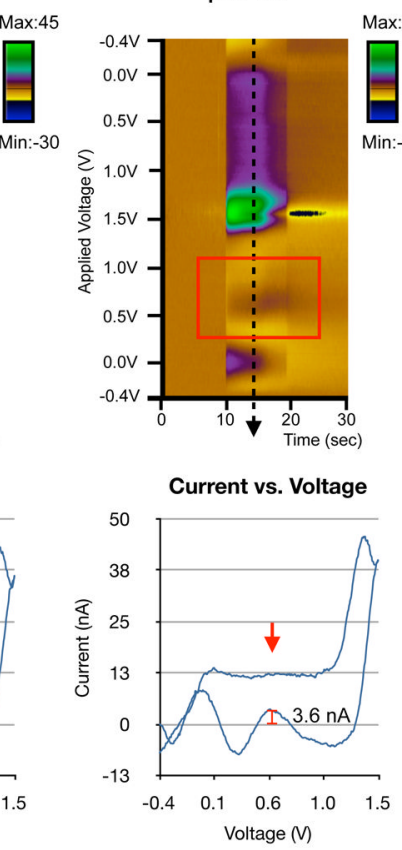

$\mathrm{pH} 7.8$
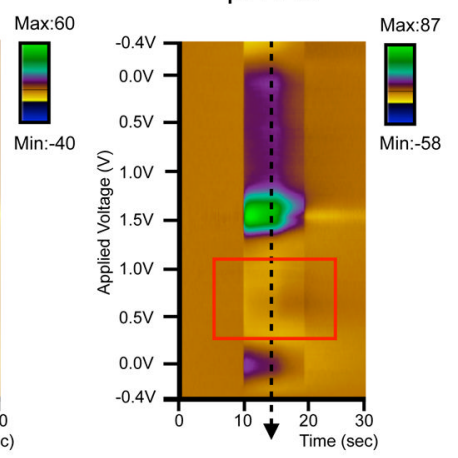

Current vs. Voltage

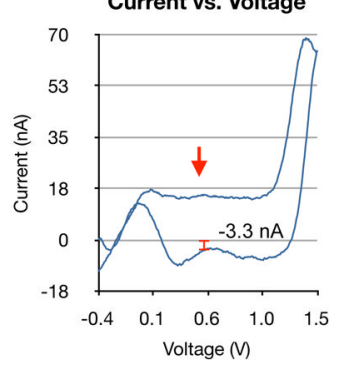

Fig 5.

(a) Color plots of DA with varying $\mathrm{pH}$ buffer in vitro via flow-cell apparatus. As the $\mathrm{pH}$ level increases, the DA oxidation peak at $0.6 \mathrm{~V}$ decreases gradually. (b) Plots of current (nA) vs. voltage (V) comparing the current measurement of the oxidation peak (indicated by the red arrows) at $0.6 \mathrm{~V}$ as the $\mathrm{pH}$ level increases. 

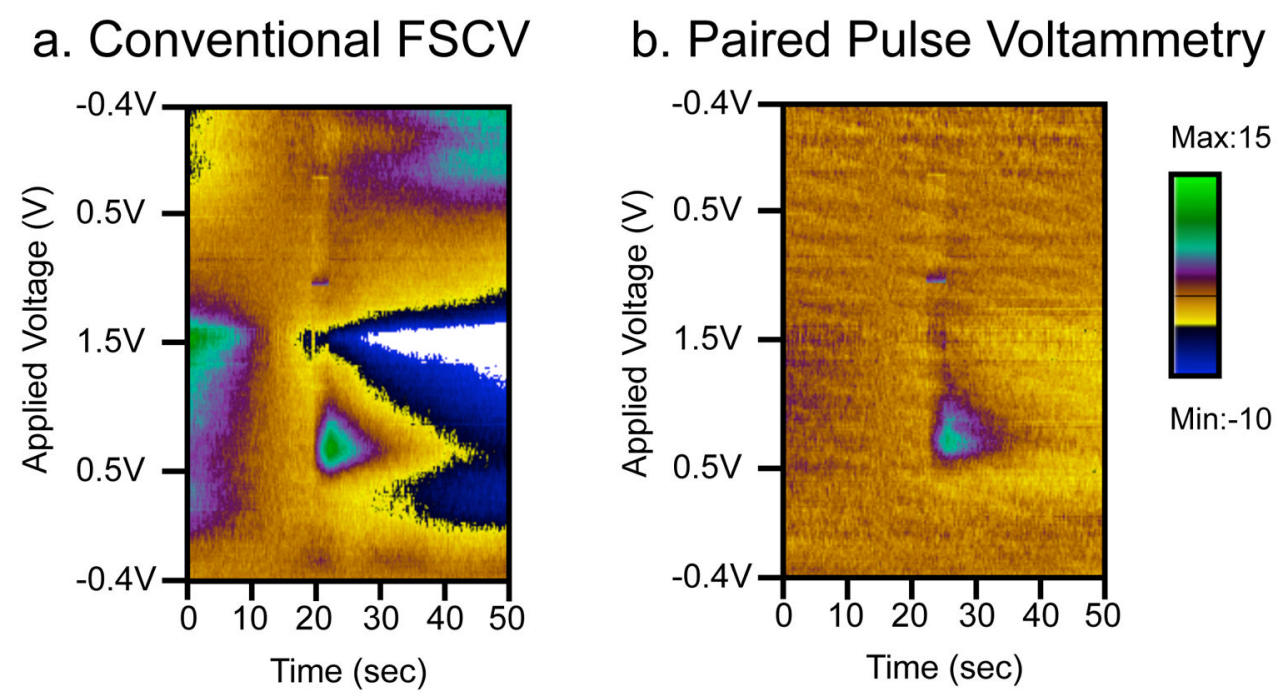

Fig 6.

PPV minimizes the background drifting effect observed during the initiation of conventional FSCV in vivo. (a) Pseudo-color plot of DA detection with the conventional FSCV method measured concurrently with background drifting. (b) Pseudo-color plot of DA detection with PPV measured concurrently with background drifting. 